\title{
Transfer RNA gene arrangement and codon usage in vertebrate mitochondrial genomes: a new insight into gene order conservation
}

\author{
Takashi P Satoh ${ }^{1 \dagger}$, Yukuto Sato ${ }^{2 \dagger}$, Naoharu Masuyama ${ }^{3,4}$, Masaki Miya $^{5}$, Mutsumi Nishida ${ }^{3 *}$
}

\begin{abstract}
Background: Mitochondrial (mt) gene arrangement has been highly conserved among vertebrates from jawless fishes to mammals for more than 500 million years. It remains unclear, however, whether such long-term persistence is a consequence of some constraints on the gene order.

Results: Based on the analysis of codon usage and tRNA gene positions, we suggest that tRNA gene order of the typical vertebrate mt-genomes may be important for their translational efficiency. The vertebrate mt-genome encodes 2 rRNA, 22 tRNA, and 13 transmembrane proteins consisting mainly of hydrophobic domains. We found that the tRNA genes specifying the hydrophobic residues were positioned close to the control region (CR), where the transcription efficiency is estimated to be relatively high. Using 47 vertebrate $\mathrm{mt}$-genome sequences representing jawless fishes to mammals, we further found a correlation between codon usage and tRNA gene positions, implying that highly-used tRNA genes are located close to the CR. In addition, an analysis considering the asymmetric nature of mtDNA replication suggested that the tRNA loci that remain in single-strand for a longer time tend to have more guanine and thymine not suffering deamination mutations in their anticodon sites.

Conclusions: Our analyses imply the existence of translational constraint acting on the vertebrate mt-gene arrangement. Such translational constraint, together with the deamination-related constraint, may have contributed to long-term maintenance of gene order.
\end{abstract}

\section{Background}

The animal mitochondrial (mt)-genome generally encodes 13 protein, 2 rRNA, and 22 tRNA genes. Although their arrangement is rather variable among invertebrate mt-genomes, a typical gene arrangement has been highly conserved among vertebrate mt-genomes from jawless fishes to mammals with some exceptions [1,2]. This implies an extremely long-term persistence of mt-gene order probably for $>500$ million years across diverse clades of vertebrates. However, it has been unclear whether such high-conservation of gene order is a consequence of some constraints, or whether it results only by sharing a common ancestry.

\footnotetext{
* Correspondence: mnishida@aori.u-tokyo.ac.jp

+ Contributed equally

${ }^{3}$ Department of Marine Bioscience, Atmosphere and Ocean Research Institute, The University of Tokyo, 5-1-5 Kashiwanoha, Kashiwa-shi, Chiba 2778564, Japan

Full list of author information is available at the end of the article

This has been a long-standing enigma for more than 20 years since the initial reports of the whole mt-genome sequence of vertebrates [3].

To address this problem, we analyzed codon usage and tRNA gene arrangements of the vertebrate mt-genomes to examine possible constraints on the gene order of vertebrate mt-genomes.

\section{Results and Discussion}

Amino acid usage and tRNA gene arrangement

We began by focusing on the fact that all of the 13 proteins encoded by the vertebrate mt-genomes are transmembrane proteins [3], which are rich in hydrophobic amino acid residues (Fig. 1). The frequency of codon usage for hydrophobic amino acids is, consequently, higher in the vertebrate mt-genome $(0.624$, the mtREV matrix [4]) compared to a general nuclear genome (0.490, the JTT matrix [5]). Based on this observation, we divided the mt-tRNA genes into two groups 


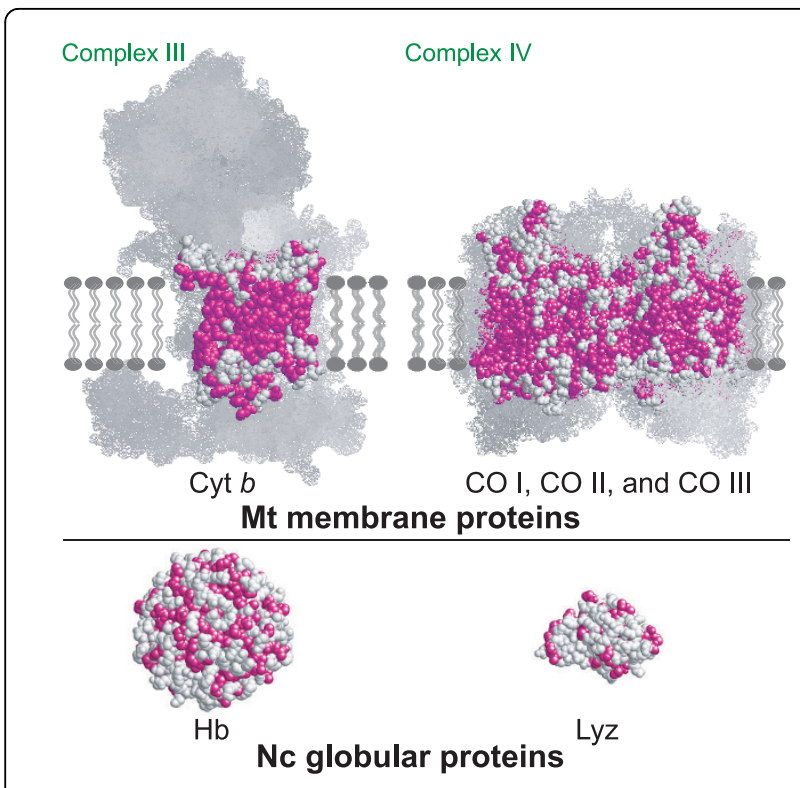

Figure 1 Spatial distribution of hydrophobic residues in the representative proteins encoded by vertebrate mitochondrial (mt) and nuclear (nc) genomes. Hydrophobic residues (Phe, Val, Leu, lle, Met, Trp, Ala, Gly, and Pro) are colored magenta. The upper panels show transmembrane proteins encoded by mt-genomes: bovine cytochrome bc complex (complex III) (left; Protein Data Bank ID [PDB]: 1SQQ) and cytochrome c oxidase complex (complex IV) (light; PDB: 1V55). In these panels, the protein subunits encoded by mt-genes are shown by space-filling models, and the remaining subunits encoded by nc genes are shown by Van der Waals' surface dot models. The lower panels show globular proteins encoded by nc-genomes: human hemoglobin A (PDB: 1BZ0) and lysozyme (PDB: 133L). The $3 \mathrm{D}$ graphical models were generated and processed using the program RasMol [30].

according to the hydrophobicity of their corresponding amino acids, and then examined their positions in the vertebrate mt-genomes (Fig. 2). This revealed that genes for tRNAs that specify hydrophobic amino acids (Fig. 2, colored magenta) are located close to the control region (CR; [6]) compared with the other genes for tRNAs specifying hydrophilic amino acids (Fig. 2, colored blue; $p=0.0295$, Mann-Whitney $U$-test, $U=27.000, n_{1}=10$, $n_{2}=12$; the detailed data based on human mt-genome is shown in Table 1).
Table 1 Comparison of the positions of mitochondrial (mt) tRNA genes corresponding to hydrophobic and hydrophilic amino acids based on human mt-genome data

\begin{tabular}{|c|c|c|c|c|c|}
\hline \multicolumn{3}{|c|}{ Hydrophobic group } & \multicolumn{3}{|c|}{ Hydrophilic group } \\
\hline tRNA & Strand $^{a}$ & Distance $^{b}$ & tRNA & Strand $^{a}$ & Distance $^{b}$ \\
\hline Phe & $\mathrm{H}$ & 1 & Glu & $L$ & 1282 \\
\hline Pro & L & 1 & Asp & $\mathrm{H}$ & 6941 \\
\hline Val & $\mathrm{H}$ & 1026 & Lys & H & 7718 \\
\hline Leu (UUR) & $\mathrm{H}$ & 2653 & Ser (UCN) & L & 8508 \\
\hline lle & $\mathrm{H}$ & 3686 & $\operatorname{Arg}$ & $\mathrm{H}$ & 9828 \\
\hline Met & $\mathrm{H}$ & 3825 & Tyr & L & 10133 \\
\hline Trp & $\mathrm{H}$ & 4935 & Cys & L & 10198 \\
\hline Gly & $\mathrm{H}$ & 9414 & Asn & L & 10295 \\
\hline Ala & L & 10369 & His & $\mathrm{H}$ & 11561 \\
\hline \multirow[t]{3}{*}{ Leu (CUN) } & $\mathrm{H}$ & 11689 & Gln & L & 11624 \\
\hline & & & $\operatorname{Ser}(A G Y)$ & $\mathrm{H}$ & 11630 \\
\hline & & & Thr & $\mathrm{H}$ & 15311 \\
\hline
\end{tabular}

a The mtDNA strand on which the tRNA gene occurs: $H$, heavy strand; $L$, light strand.

${ }^{\mathrm{b}}$ Base-pair distance between the 3 '-end of control region and the 5 '-end of each tRNA gene.

Such tRNA gene localization may indicate that, in the typical vertebrate mt-genomes, highly-used tRNA genes are located in the genomic region close to the $\mathrm{CR}$, where the transcription efficiency is thought to be relatively high. The transcription of the vertebrate mt-genome is initiated from regulatory elements within the CR [6,7], and thus, the complete transcription of the genes into mRNA and functional RNAs would be more successful in the genomic region closer to the CR. In fact, the two rRNA genes immediately adjacent to the CR (12 S and 16S; see Fig. 2) are highly expressed [8]. Likewise, the tRNA genes localized close to the CR, which specify hydrophobic residues, would also be highly expressed. Such an efficient production of the highly-used tRNAs may be favorable for translation of vertebrate mt-genomes.

\section{Correlation between codon usage and tRNA gene position}

Given the increasing availability of full-length mt-genome sequence data from a broad range of vertebrate

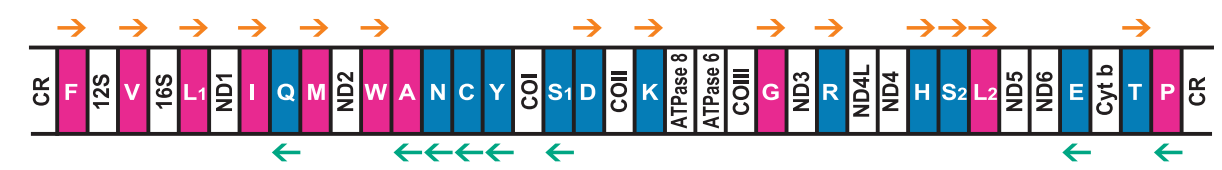

Figure 2 Schematic diagram outlining the typical gene order in the vertebrate mt-genome. The tRNA genes (designated using singleletter amino acid codes) that specify hydrophobic and hydrophilic amino acids are colored magenta and blue, respectively. Orange and green arrows show the transcriptional orientation of each tRNA gene on the heavy $(\mathrm{H})$-strand and light $(\mathrm{L})$-strand, respectively. CR, the control region; $12 \mathrm{~S}$ and $16 \mathrm{~S}$, the $12 \mathrm{~S}$ and $16 \mathrm{~S}$ ribosomal RNA genes; ND1-6, and 4L, NADH dehydrogenase subunits 1-6 and the 4L gene; COI-III, cytochrome c oxidase subunits I-III genes; ATPase 6 and 8 , ATPase subunits 6 and 8 genes; Cyt $b$, the cytochrome $b$ gene; $L_{1}$ and $L_{2}$ indicate the tRNA-Leu (UUR) and tRNA-Leu (CUN) genes, respectively; $S_{1}$ and $S_{2}$, the tRNA-Ser (UCN) and tRNA-Ser (AGY) genes. 
species, the tRNA gene arrangement with regards to amino acid usage can be assessed more quantitatively by analyzing a correlation between physical (base pair [bp]) distance from the CR to each tRNA gene and codon usage of the mt-genome. We analyzed correlation between these two parameters using both parametric and non-parametric methods based on the mt-genome sequences of 47 representative vertebrates from jawless fish, cartilaginous fish, ray-finned fish, amphibians, reptiles, birds, and mammals (species names are listed in Table 2). Among these 47 species, 33 represent the "evolutionarily stable" mt-gene orders including the typical gene arrangement of vertebrates and slightly rearranged gene orders of lamprey and birds (supplementary Fig. S1 [see Additional file 1]). The remaining 14 species from ray-finned fish, amphibians, and reptiles represent rearranged gene orders within these lower taxa. More rearranged $\mathrm{mt}$-genomes were sampled from ray-finned fish (8 species) than from amphibians and reptiles (6 species) because ray-finned fish is a sister group to all tetrapods, and shows greater diversity in terms of mt-gene rearrangements.

In the correlation analysis, we aimed to eliminate the effects of shared common ancestry [9] based on independent contrast analysis [10] of the codon usage and tRNA positions. Independent contrasts for these two variables were estimated using the program CAIC [11] based on a composite tree of the sampled species that was constructed from recent molecular phylogenies for the major clades of vertebrates (supplementary Fig. S2 [see Additional file 1]). This method focuses on differences only between sister lineages or nodes in a phylogeny, which have arisen after a split, therefore yielding sets of independent "contrasts" [10]. Our data points were derived from ancestral states (namely, various nodes across the tree) based on independent contrasts of tRNA genes (listed in supplementary Table S1, Table S2, and Table S3 [see Additional file 1]). Thus, many data points were above the level of major clades such as mammals, birds, reptiles, amphibians, actinopterygians, chondrichthyans, and agnathans, and the total number of data points became much smaller than the number of pair-wise comparisons.

As a result, we found a significant correlation between the tRNA positions and codon usage (Fig. 3; evolutionarily stable gene orders: Pearson's correlation coefficient $r=-0.1260$, one-tailed $p=0.0104, n=336$; Spearman rank-correlation coefficient $r_{\mathrm{s}}=-0.0827, t=-2.2171$, one-tailed $p=0.0136, n=336$, d.f. $=334$; rearranged gene orders within lower taxa: Pearson's correlation coefficient $r=-0.2209$, one-tailed $p=0.0001, n=308$; Spearman rank-correlation coefficient $r_{\mathrm{s}}=-0.2336$,
Table 2 List of the species studied and the DDBJ/EMBL/ GenBank accession numbers of their mitochondrial genome sequences

Scientific name Common name Accession No.

Evolutionarily stable gene orders

Agnatha

Eptatretus burgeri

Inshore hagfish

AJ278504

Myxine glutinosa

Atlantic hagfish

AJ404477

Petromyzon marinus

Sea lamprey

U11880

Chondrichthyes

Chimaera monstrosa

Rabbitfish

Scyliorhinus canicula

Catshark

AJ310140

Squalus acanthias

Spiny dogfish

Y16067

Heterodontus francisci

Horn shark

Raja radiata

Thorny skate

Y18134

AJ310141

AF106038

Actinopterygii

Lepisosteus spatula

Alligator gar

AP004355

Cyprinus carpio

Common carp

AP009047

Gadus morhua

Atlantic cod

X99772

Pagrus major

Seabream

AP002949

Paralichthys olivaceus

Bastard halibut

AB028664

Amphibia

Ambystoma mexicanum

Andrias japonicus

Axolotl

AJ584639

Bombina bombina

Giant salamander

AB208679

Xenopus laevis

Fire-bellied toad

AY458591

Ichthyophis glutinosus

Clawed frog

M10217

AY456251

Reptilia
Chelonia mydas

Geochelone pardalis

Gekko gecko

Iguana iguana

Geocalamus acutus

Aves

Gallus gallus

Nipponia nippon

Vidua chalybeata

Falco peregrinus

Buteo buteo

Mammalia

Homo sapiens

Mus musculus

Bos taurus

Canis lupus

Balaenoptera musculus
Seaturtle

Tortoise

Tokay

Iguana

Worm lizard

AB012104

DQ080041

AY282753

AJ278511

AY605476

$\times 52392$

AB104902

AF090341

AF090338

AF380305 
Table 2 List of the species studied and the DDBJ/EMBL/ GenBank accession numbers of their mitochondrial genome sequences (Continued)

\begin{tabular}{|c|c|c|}
\hline \multicolumn{3}{|c|}{ Rearranged gene orders within lower taxa } \\
\hline \multicolumn{3}{|l|}{ Actinopterygii } \\
\hline Eurypharynx pelecanoides & Pelican eel & AB046473 \\
\hline Saccopharynx lavenbergi & Gulper eel & AB047825 \\
\hline Gonostoma gracile & Slender fangjaw & AB016274 \\
\hline Myctophum affine & Lantern fish & AP002922 \\
\hline Caelorinchus kishinouyei & Grenadier & AP002929 \\
\hline Aspasma minima & Clingfish & AP004453 \\
\hline Aulostomus chinensis & Trumpet fish & AP009197 \\
\hline Chlorurus sordidus & Parrot fish & AP006567 \\
\hline \multicolumn{3}{|l|}{ Amphibia } \\
\hline Rana nigromaculata & Pond frog & AB043889 \\
\hline Buergeria buergeri & Kajika frog & AB127977 \\
\hline Rhacophorus schlegelii & Tree frog & AB202078 \\
\hline \multicolumn{3}{|l|}{ Reptilia } \\
\hline Boa constrictor & Boa & AB177354 \\
\hline Dinodon semicarinatus & Colubrid snake & AB008539 \\
\hline Gloydius blomhoffii & Pit viper & EU913477 \\
\hline
\end{tabular}

$t=-3.9727$, one-tailed $p<0.0001, n=308$, d.f. $=306$; all of the $47 \mathrm{mt}$-genomes: Pearson's correlation coefficient $r$ $=-0.1643$, one-tailed $p<0.0001, n=990$; Spearman rank-correlation coefficient $r_{\mathrm{s}}=-0.1441, t=-4.4827$, one-tailed $p<0.0001, n=990$, d.f. $=988$ ). These results imply that the tRNA genes that correspond to highlyused codons were located nearer the CR compared with the others. The data points for the tRNA-Leu (CUN) and tRNA-Thr genes, however, deviated visibly from the trend. The usage of their corresponding codons was relatively high, although their genomic positions were distant from their putative transcription start site in the CR (see Fig. 3). When these two genes were excluded, the correlation became stronger (not shown as figures; evolutionarily stable gene orders: $r=-0.4207$, one-tailed $p<0.0001, n=304$; rearranged gene orders within lower taxa: $r=-0.2760$, one-tailed $p<0.0001, n=294$; all of the $47 \mathrm{mt}$-genomes: $r=-0.4121$, one-tailed $p<$ $0.0001, n=900$ ).

The significance of the above correlations, however, may be due to the larger number of degrees of freedom generated by multiple-species comparisons on multiple tRNA genes, although we sought to eliminate the effect of shared common ancestry as described above. To limit this effect and to corroborate our results, we took two other approaches: first, we averaged codon usage and distance from the CR for each of 22 tRNA across taxa, respectively, yielding 22 independent data points of 22 non- homologous tRNA genes. This set of data might reflect an ancient adaptation between codon usage and tRNA positions of an original vertebrate mt-genome. Second, we analyzed variations of codon usage and distance from the CR of each of the 22 tRNA genes across taxa, specifically focusing on the rearranged gene orders within lower taxa. The second analysis might detect a recent adaptation between codon usage and tRNA positions in taxonomic groups concerned (lower than an order level; for details, see supplementary Fig. S2 [see Additional file 1]).

By way of the first approach, we found a weak (but statistically not significant) correlation between mean codon usage and tRNA positions (Fig. 4; evolutionarily stable gene orders: $r=-0.1440$, one-tailed $p=0.2613, n$ $=22$; rearranged gene orders within lower taxa: $r=$ -0.2345 , one-tailed $p=0.1468, n=22$; all of the $47 \mathrm{mt}-$ genomes: $r=-0.1719$, one-tailed $p=0.2222, n=22$ ), implying that the tRNA genes corresponding to highlyused codons were located nearer the CR. This relationship became stronger and the correlation significant when the outlier tRNA-Leu (CUN) and tRNA-Thr genes were excluded (not shown as figures; evolutionarily stable gene orders: $r=-0.4510$, one-tailed $p=0.0230, n$ $=20$; rearranged gene orders within lower taxa: $r=$ -0.4206 , one-tailed $p=0.0324, n=20$; all of the $47 \mathrm{mt}$ genomes: $r=-0.4503$, one-tailed $p=0.0232, n=20$ ). These results would support the correspondence between codon usage and mt-tRNA positions possibly in the origin of the vertebrate mt-genome gene order, although such constraint might have been weak.

The second approach involving the meta-analysis of the results of the respective 22 tRNA gene sets showed that the rearranged gene orders within lower taxa had little effect on the preexisting correlation between codon usage and tRNA positions (an overall weighted Fisher's $r$ $=0.0722$, Stouffer's combined $p=0.3128$; the results of the correlation in the respective 22 tRNAs are shown in the supplementary Fig. S3 and Fig. S4 [see Additional file 1]). This result can be explained as a result of elimination of novel gene arrangement deviating from the relationship. In fact, among 13 of phylogenetically independent cases of gene-order rearrangements (supplementary Fig. S5 [see Additional file 1]), three cases (deep-sea eels, frogs, and Tuatara) were observed to have improved correlation between tRNA position and codon usage compared to the evolutionarily stable gene orders; majority of the cases of gene order rearrangements showed no improvement in the relationship (detailed data are shown in supplementary Table S4 [see Additional file 1]). This implies that not a few gene order rearrangements without improvement have existed for some periods of evolutionary time. It is noted, however, that the correlation between tRNA position and codon usage was significant (or marginally significant) 


\section{A Evolutionarily stable gene orders (33 species)}

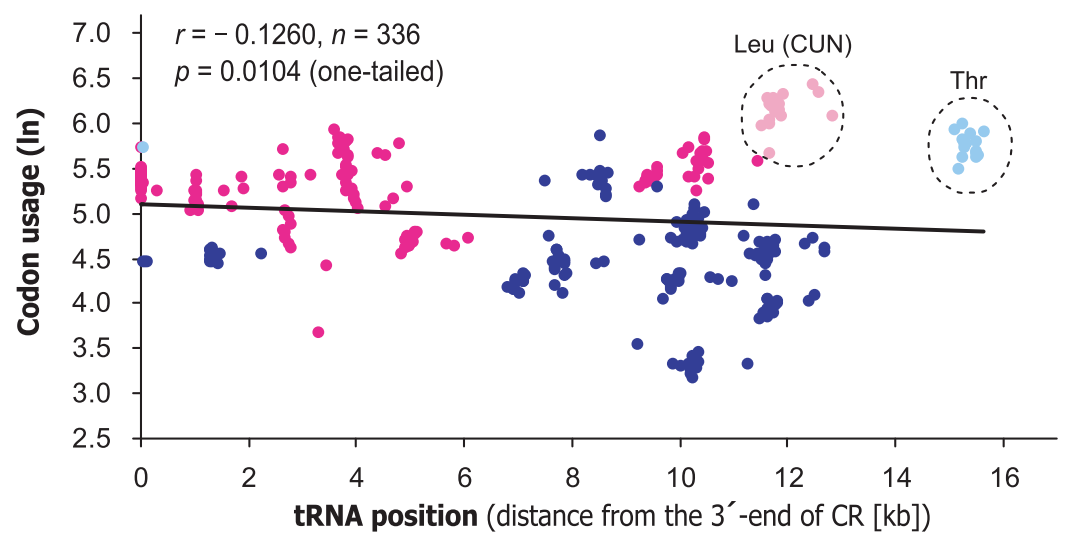

B Rearranged gene orders within lower taxa (14 species)

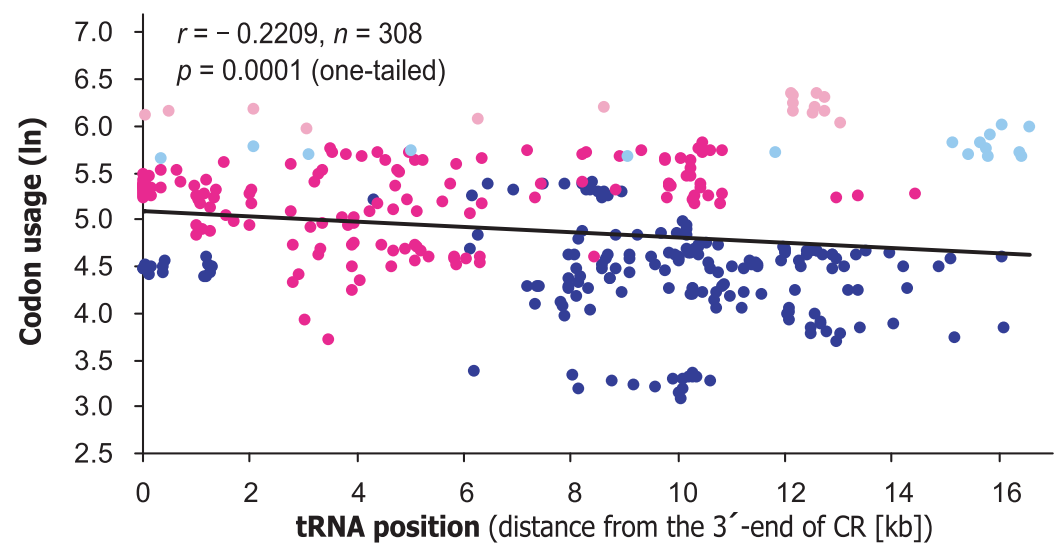

C All gene orders used (47 species)

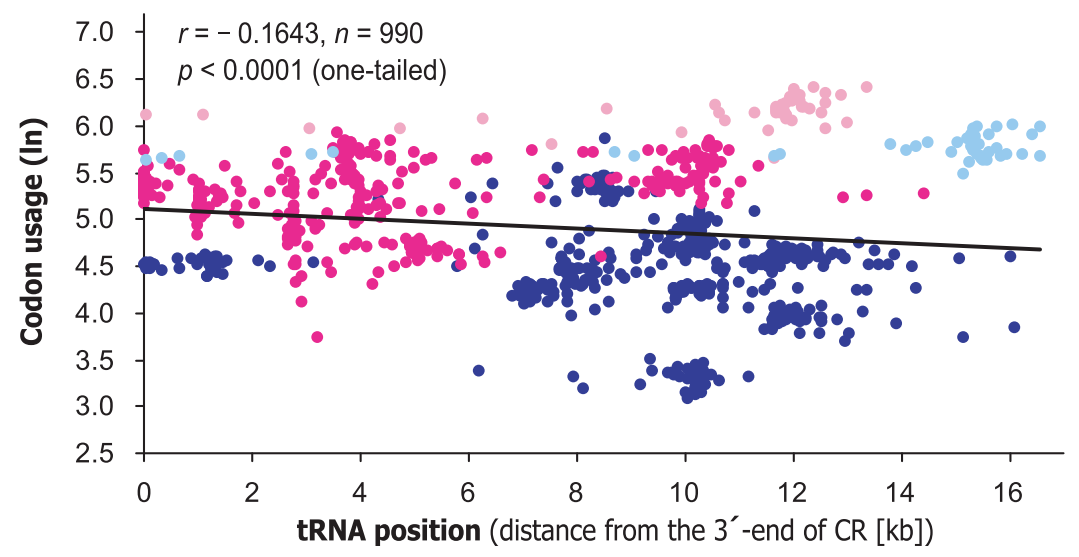

Figure 3 A linear regression plot between position of tRNA genes and usage of the corresponding codon. The data points were derived from an independent contrasts analysis using the program CAIC [11] based on vertebrate mt-genome sequences from (A) 33 species with evolutionarily stable gene order, (B) 14 species with rearranged gene orders within lower taxa, and (C) all of the 47 species selected (Table 2) and recent molecular phylogenies for vertebrates (supplementary Fig. S2 [see Additional file 1]). Data points for the tRNA genes that specify hydrophobic and hydrophilic amino acids are colored magenta and blue, respectively; exceptionally, the data points for the tRNA-Leu (CUN) and tRNA-Thr are colored light pink and light blue, respectively. The regression lines were derived from the all data points in each plot. When the tRNA genes of $\mathrm{H}$-strand and L-strand were analyzed separately, the correlation remained negative for either of the strands (data not shown). 


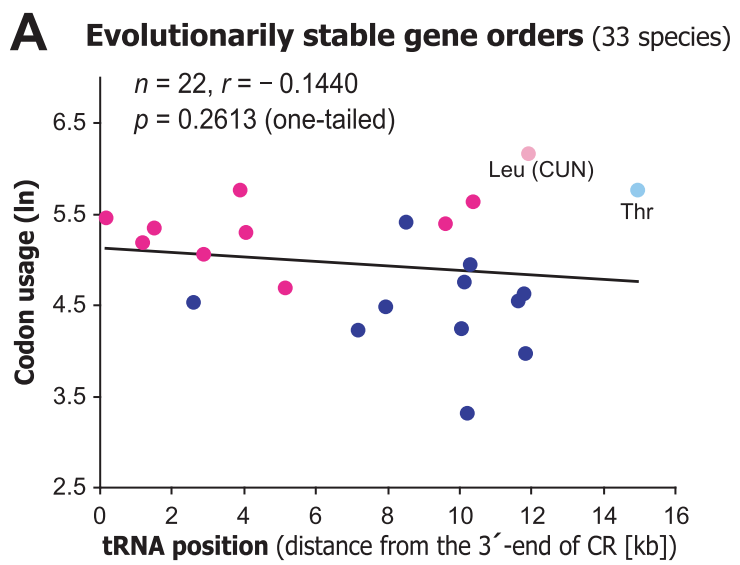

B Rearranged gene orders within lower taxa (14 species)

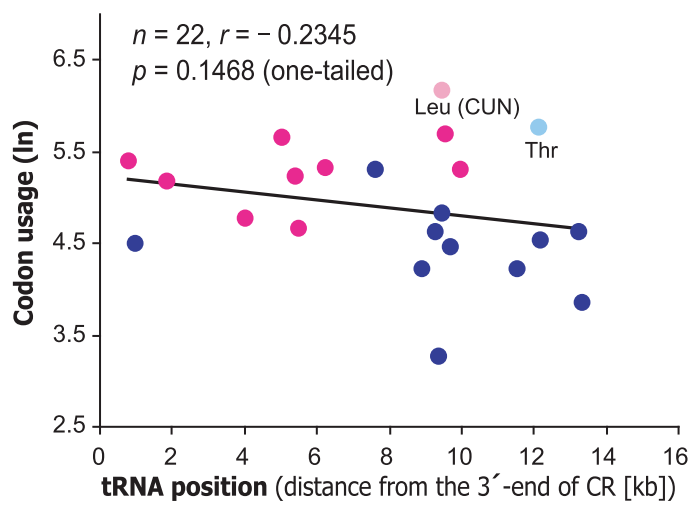

C All gene orders used (47 species)

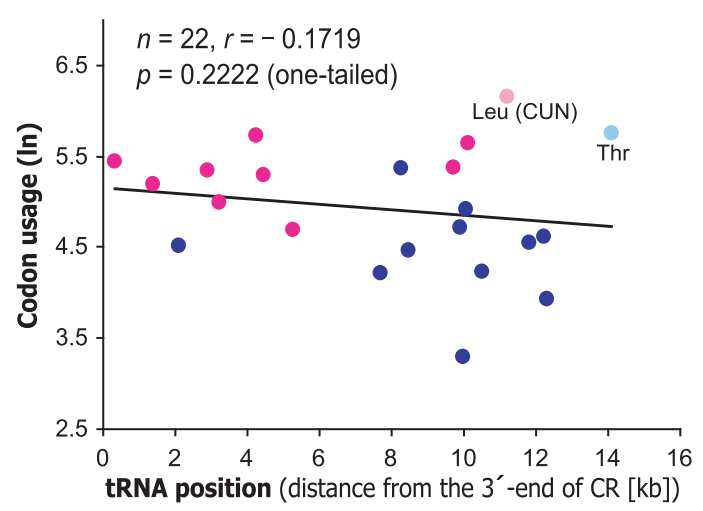

Figure $4 \mathrm{~A}$ linear regression plot between mean position of the tRNA genes and mean usage of the corresponding codon. The data points represent the average of each of tRNA across the mt-genome sequences from (A) 33 species with evolutionarily stable gene order, (B) 14 species with rearranged gene orders within lower taxa, and (C) all of the 47 species selected (Table 2). Data points for the tRNA genes that specify hydrophobic and hydrophilic amino acids are colored magenta and blue, respectively; exceptionally, the data points for the tRNA-Leu (CUN) and tRNA-Thr are colored light pink and light blue, respectively. The regression lines were derived from the all data points in the plot. for the above three cases only. Their novel gene order arrangements might be maintained through some forms of natural selection.

On the basis of these sets of analyses, we propose that the tRNA gene arrangement of vertebrate mt-genomes, and possibly that of an ancestral, original vertebrate mtgenome, may be adaptive with regard to translational efficiency. The genes close to the CR, where the transcription initiation sites of both strands exist, appear to be highly expressed in the vertebrate mt-genomes [8]. Consequently, genes of tRNAs specifying highly-used codons would be favorably located close to the CR to ensure the efficient translation of the protein-coding genes in the vertebrate mt-genomes.

\section{Mitochondrial gene arrangement and translational constraint}

On the basis of the results obtained from our analyses, we suggest the existence of translational constraint on the positions of mt-tRNA genes, but not on their gene copy numbers, in the vertebrate mt-genomes, although the constraint may be weak. In nuclear genomes, translational selection is known to promote adaptation of tRNA gene number to the usage of the corresponding codon $[12,13]$. Clear association of tRNA gene number with codon usage has been observed in the genomes of various organisms ranging from $E$. coli to humans [14-19]. The vertebrate mt-genome is also likely exposed to translational selection because vertebrates are considered to be metabolically active and have high rates of ATP synthesis. However, translational selection would not act at the level of tRNA gene numbers in the vertebrate $\mathrm{mt}$-genome since it is extremely compact and the number of contained genes is limited.

Recently, some studies suggest the replication and translational constraints affected the positions of translational genes such as RNA polymerase, rRNA, and tRNA genes in bacterial genomes [20], and abundant and broadly expressed genes in the human genome [21]. Such constraints associated with translation and gene expression may also have limited the gene order rearrangement of vertebrate $\mathrm{mt}$-genomes, specifically the rearrangements which interfere with transcriptional efficiency of mt-tRNAs (see Fig. 3B, Fig. 4B, and supplementary Fig. S3 and Fig. S4 [see Additional file 1]). This constraint may have driven the conservation of the mtgene arrangement among vertebrates from jawless fishes to mammals for more than 500 million years.

Gene-order rearrangements are often found in vertebrate mt-genomes within lower taxonomic categories such as families, genera, and species $(166 / 769=21.6 \%$ of species [22]), however, there are no extensive rearrangements shared across higher taxa, which are likely to have persisted for long evolutionary periods of 
time [1]. This observation further implies the existence of constraint on vertebrate mt-gene orders, possibly through translational efficiency as discussed above. Exceptionally, mt-genomes of birds and lampreys show some little deviation from the typical gene order; either of the bird or lamprey have mt-genomes showing some changes in tRNA-Glu, tRNA-Thr, and tRNA-Pro gene positions (see supplementary Fig. S1 [see Additional file 1]). These non-typical gene orderings, however, interfere little with the correlation reported above (when birds and lampreys were excluded; $r=-0.1499$, one-tailed $p=0.0071, n=267$ ). This also implies that the deviations of the tRNA-Leu (CUN) and tRNA-Thr from the supported relationship (see Fig. 3) do not arise from the rearrangements of birds and lampreys.

We found two tRNA genes that contradict our notion. The tRNA-Leu (CUN) and tRNA-Thr genes were located distant from their putative transcription start site in the CR, but the usage of their corresponding codons was relatively high (Fig. 3 and Fig. 4). The presence of such outlier tRNAs is not strange because mttRNA genes are also under constraints associated with their other functions such as punctuation markers during the pre-mRNA processing of adjacent genes [7]. However, one attractive possible constraint on mt-tRNA position is deamination gradients during replication and possibly transcription of the mt-DNA [23]. Deamination commonly occurs in single-stranded DNA exposed during replication or transcription, causing mutations from adenine to guanine and from cytosine to thymine $[23,24]$. Consequently, the gene loci exposed in single strand for longer time in replication and transcription are more prone to suffer these deamination mutations. In the replication of mtDNA $[25,26]$, the Heavy $(\mathrm{H})$ strand is initially replicated from the $\mathrm{H}$-strand replication origin $\left(\mathrm{O}_{\mathrm{H}}\right)$ in the CR. The parental, original $\mathrm{H}$-strand is exposed as a single strand (see right part of the Fig. 5A, gray line). Subsequently, using this original $\mathrm{H}$-strand as a lagging strand, the Light (L)-strand is replicated from the $\mathrm{L}$-strand replication origin $\left(\mathrm{O}_{\mathrm{L}}\right)$ in a WANCY region [26] (Fig. 5A, green arrow). Based on this view, the tRNA genes located more distant from the $\mathrm{O}_{\mathrm{L}}$ along the direction of L-strand replication would be exposed as a single strand for a longer time. Consequently, such tRNA genes are more likely to undergo the deamination mutations. A similar deamination gradient might also exist in transcription initiated from the CR [23].

The present analysis considering the asymmetric nature of mtDNA replication provided results match with the above prediction (Fig. 5). The tRNA loci that are expected to be exposed as a single strand for a longer time tend to have more guanine $(\mathrm{G})$ and thymine $(\mathrm{T})$ in their anticodon region on the coding strands (Fig. 5A: tRNA loci located between the $\mathrm{O}_{\mathrm{L}}$ and $\mathrm{O}_{\mathrm{H}}$ along the direction of L-strand replication: $r=0.1750$ one-tailed $p=0.2838, n=13$; Fig. 5B: all tRNA genes: $r=0.4281$ one-tailed $p=0.0234, n=22$ ). This suggests that the tRNA gene arrangement of typical vertebrate mt-genome is also adaptive in avoiding mutations in anticodons through deamination during replication, and possibly, in transcription [23]. Regarding the correlation coefficients, the deamination-related constraint may be stronger than the codon/amino-acid usage-related constraint discussed above.

\section{Conclusion}

In this paper, we propose that the high conservation of the gene arrangement of the vertebrate mt-genome is underpinned not only by a shared common ancestry, but also by translational constraint acting on the tRNA gene arrangement. This conclusion can be derived from the simple observation that the mt-tRNA genes corresponding to hydrophobic amino acids, which are frequently used in translation of the mt-genes, are localized close to the CR. In addition, an analysis considering the asymmetric nature of mtDNA replication suggested that deamination-related constraint against mutations in tRNA anticodons is also an important determinant of the tRNA gene arrangement in the typical vertebrate mt-genome. The translational constraint together with the deamination-related constraint may have contributed to shaping and maintaining the typical gene order of the vertebrate mt-genomes.

\section{Methods}

\section{Taxonomic sampling of mitochondrial genome data}

To consider variation in codon usage and gene arrangement across typical vertebrate mt-genomes, we chose five species from each of mammals, birds, reptiles, amphibians, actinopterygians, and chondrichthyans, and three species from agnathans, for which only few mtgenome sequences were available in databases. Those 33 mt-genomes are defined as "evolutionarily stable" gene orders. In addition, to include mt-genomes that have rearranged gene orders within lower taxa, we chose eight species from actinopterygians and three species from reptiles and amphibians, respectively. Those 14 mt-genomes are defined as "rearranged gene orders within lower taxa". Species names and GenBank accession numbers of the mt-genomes are listed in Table 2: these species were selected to represent a broad niche breadth. The invertebrates could not be analyzed in this study, because a transcription system of the mt-genome and a sound phylogenetic framework are unclear for most of them. 

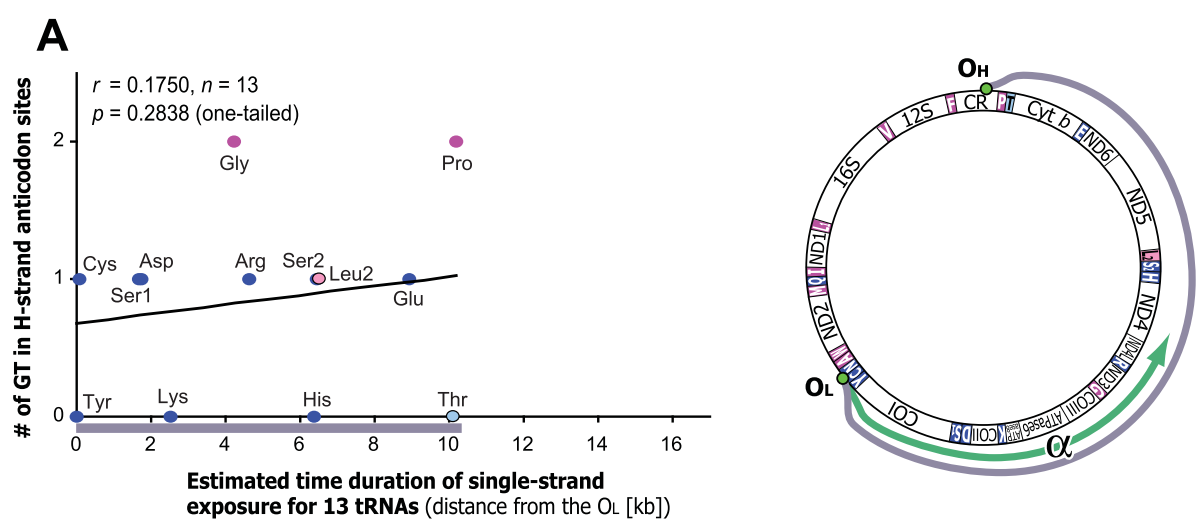

B
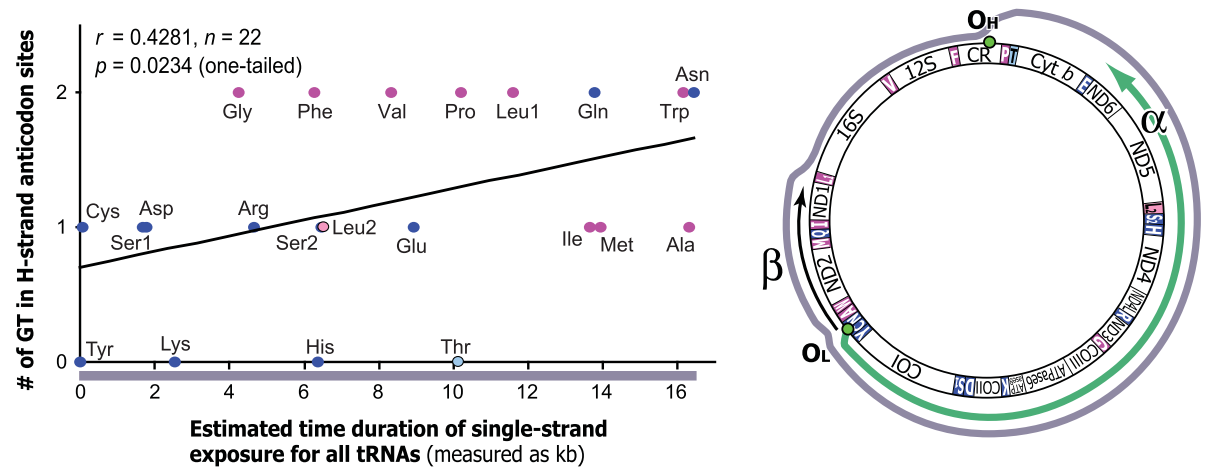

Figure 5 Correlation between the number of guanine (G) and thymine (T) in 1st and 2nd anticodon positions of tRNA loci and expected time duration of single-strand exposure during mtDNA replication. The analysis is based on a human mt-genome. Data points for the tRNA genes that specify hydrophobic and hydrophilic amino acids are colored magenta and blue, respectively; exceptionally, tRNA-Leu $(C U N)$ and tRNA-Thr are shown in light pink and light blue, respectively. (A) Analysis for tRNA genes located between the $O_{L}$ and $O_{H}$ along the direction of L-strand replication (tRNA-Cys, -Tyr, -Ser1, -Asp, -Lys, -Gly, -Arg, -His, -Ser2, -Leu2, -Glu, -Thr, and -Pro). The expected time duration of single-strand exposure was measured as distance from the $\mathrm{O}_{\mathrm{L}}$ along the direction of $\mathrm{L}$-strand replication. This distance is named as $\alpha$, as depicted in the right part of the panel A. (B) Analysis for all tRNA genes. For the above-mentioned 13 tRNA genes (described in the legend of the panel A), the duration of single-strand exposure was measured as $\alpha$. For the other 9 tRNA genes (tRNA-Asn, -Ala, -Trp, -Met, -Gln, -lle, -Leu1, - Val, and -Phe), the duration of single-strand exposure was estimated as $(\alpha-\beta)$, where $\beta$ is distance from the $O_{L}$ along the opposite direction of $\mathrm{L}$-strand replication. This is because, among those 9 tRNA loci, the tRNAs located more distant from the $\mathrm{O}_{\mathrm{L}}$ along the opposite direction of $\mathrm{L}$ strand replication remain in double-stranded DNA for a longer time based on the displacement model of mtDNA replication [26].

\section{Measuring codon usage and the position of each tRNA gene}

The usage of each codon was counted in the sequence of the 13 protein-coding genes (ND1, ND2, ND3, ND4, ND4L, ND5, ND6, CO I, CO II, CO III, ATPase6, ATPase8, and Cyt $b$ ) of the mt-genomes examined. The overlapping codons between ATPase 8 and ATPase 6, and between ND4L and ND4 were considered once for each gene, because the open reading frame was different among these neighboring genes. To measure the position of each tRNA gene, base-pair distances from the 3' end of CR to the 5' end of each tRNA gene were counted in their respective positions on the $\mathrm{H}$ - and L-strands of mtgenome sequences. Although the accurate locations of the transcription start sites of mt-genome are unknown in most of the vertebrate species, it is assumed that the transcription start site for heavy and light strands may differ in distance from the 3' end of CR on the respective strand. Therefore, we examined whether such supposed differences affect the analysis in this study, and we found that the hypothetical differences of $\pm 150 \mathrm{bp}$ and \pm 500 bp in distance, which are based on a reference [27], do not affected the significance of the results of Mann-Whitney $U$-test and correlation analyses shown in the Results. Thus, we considered that measuring the positions of tRNA genes based on their base pair distances from the 3 ' end of the CR is justified.

\section{Regression analysis considering the effects of shared common ancestry}

To examine whether the frequency of usage of each codon varies with the position of its corresponding 
tRNA gene (the base-pair distance from the CR), we calculated Pearson's correlation coefficient $(r)$ and Spearman rank-correlation coefficient $\left(r_{\mathrm{s}}\right)$, and evaluated the significance of the relationship both parametrically and non-parametrically, respectively. To account for the effect of shared common ancestry [9], "independent contrasts" [10] for these two variables were estimated using the program CAIC [11] based on a composite tree of the sampled species (supplementary Fig. S2 [see Additional file 1]). The typical mt-gene order has predominated and persisted in most of the major vertebrate lineages for more than 500 million years, however, local gene order rearrangements and codon usage variation have been observed and described in vertebrates $[1,2,28]$. By considering such potential changeability of $\mathrm{mt}$-gene order and codon usage, we regarded the data points obtained from independent contrast analysis as virtually independent of each other, although all vertebrate mt-genomes share a common ancestor. The analysis using the program CAIC was performed using logarithmically transformed data to focus on the proportional change in the variables. The validity of this approach is discussed in the CAIC User's Guide [29].

\section{Additional material}

Additional file 1: Supplementary figures and tables. This PDF file includes supplementary figures $\mathrm{S} 1-\mathrm{S} 5$ and tables $\mathrm{S} 1-\mathrm{S} 4$.

\section{Abbreviations}

BP: base-pair; CR: control region; MT: mitochondria; TRNA: transfer RNA.

\section{Acknowledgements}

We thank our colleagues at the Atmosphere and Ocean Research Institute of the University of Tokyo for helpful discussions. This manuscript has greatly benefited from the constructive and helpful comments of two reviewers. The final version of the manuscript was carefully read by Dr. Christopher Loretz, to whom we are grateful. This study was partially supported by Grants-in-Aid from the Japan Society for the Promotion of Science to MN, MM, and YS, the NF-Hadal Environmental Science Education Program from the Nippon Foundation to TPS, the Sasakawa Scientific Research Grant from The Japan Science Society to YS, and NIG (National Institute of Genetics, Japan) postdoctoral fellowship to YS

\section{Author details \\ ${ }^{1}$ Collection Center, National Museum of Nature and Science, 3-23-1 Hyakunincho, Shinjuku-ku, Tokyo 169-0073, Japan. ${ }^{2}$ Division of Population Genetics, National Institute of Genetics, 1111 Yata, Mishima, Shizuoka 411 8540, Japan. ${ }^{3}$ Department of Marine Bioscience, Atmosphere and Ocean Research Institute, The University of Tokyo, 5-1-5 Kashiwanoha, Kashiwa-shi, Chiba 277-8564, Japan. ${ }^{4}$ Resonarch Co., Ltd., 2-2-23 Josui-shinmachi, Kodaira, Tokyo 187-0023, Japan. ${ }^{5}$ Department of Zoology, Natural History Museum and Institute, 955-2 Aoba-cho, Chuo-ku, Chiba 260-8682, Japan.}

\section{Authors' contributions}

TPS, YS, and MN designed the study. TPS, YS, and NM carried out the analyses. TPS and YS drafted the manuscript. MM and MN participated in coordination and helped to draft the manuscript. All authors read and approved the final version of the manuscript.
Received: 14 October 2009 Accepted: 19 August 2010

Published: 19 August 2010

\section{References}

1. Boore JL: Animal mitochondrial genomes. Nucleic Acids Res 1999 27:1767-1780.

2. Inoue JG, Miya M, Tsukamoto K, Nishida M: Evolution of the deep-sea gulper eel mitochondrial genomes: large-scale gene rearrangements originated within the eels. Mol Biol Evol 2003, 20:1917-1924.

3. Anderson S, Bankier AT, Barrell BG, de Bruijn MH, Coulson AR, Drouin t, Eperon IC, Nierlich DP, Roe BA, Sanger F, Schreier PH, Smith AJ, Staden R, Young IG: Sequence and organization of the human mitochondrial genome. Nature 1981, 290:457-465.

4. Adachi J, Hasegawa M: Model of amino acid substitution in proteins encoded by mitochondrial DNA. J Mol Evol 1996, 42:459-468.

5. Jones DT, Taylor WR, Thornton JM: The rapid generation of mutation data matrices from protein sequences. Comput Appl Biosci 1992, 8:275-282.

6. Chang DD, Clayton DA: Identification of primary transcriptional start sites of mouse mitochondrial DNA: accurate in vitro initiation of both heavyand light-strand transcripts. Mol Cell Biol 1986, 6:1446-1453.

7. Ojala D, Montoya J, Attardi G: tRNA punctuation model of RNA processing in human mitochondria. Nature 1981, 290:470-474.

8. Christianson TW, Clayton DA: A tridecamer DNA sequence supports human mitochondrial RNA 3'-end formation in vitro. Mol Cell Biol 1998, 8:4502-4509.

9. Harvey PH, Pagel MD: The Comparative Method in Evolutionary Biology Oxford: Oxford University Press 1991.

10. Felsenstein J: Phylogenies and the comparative method. Am Nat 1985 , 125:1-15.

11. Purvis $A$, Rambaut $A:$ Comparative analysis by independent contrasts (CAIC): an Apple Macintosh application for analysing comparative data. Comput Appl Biosci 1995, 11:247-251.

12. Akashi H: Gene expression and molecular evolution. Curr Opin Genet Dev 2001, 11:660-666.

13. Akashi H: Translational selection and yeast proteome evolution. Genetics 2003, 164:1291-1303.

14. Ikemura T: Correlation between the abundance of Escherichia coli transfer RNAs and the occurrence of the respective codons in its protein genes: a proposal for a synonymous codon choice that is optimal for the E. coli translational system. J Mol Biol 1981, 146:1-21.

15. Yamao F, Andachi Y, Muto A, Ikemura T, Osawa S: Levels of tRNAs in bacterial cells as affected by amino acid usage in proteins. Nucleic Acids Res 1991, 19:6119-6122.

16. Moriyama EN, Powell JR: Codon usage bias and tRNA abundance in Drosophila. J Mol Evol 1997, 45:514-523.

17. Percudani R, Pavesi A, Ottonello S: Transfer RNA gene redundancy and translational selection in Saccharomyces cerevisiae. J Mol Biol 1997, 268:322-330.

18. Duret L: tRNA gene number and codon usage in the $C$. elegans genome are co-adapted for optimal translation of highly expressed genes. Trends Genet 2000, 16:287-289.

19. Kotlar D, Lavner $Y$ : The action of selection on codon bias in the human genome is related to frequency, complexity, and chronology of amino acids. BMC Genomics 2006, 7:67.

20. Couturier E, Rocha EP: Replication-associated gene dosage effects shape the genomes of fast-growing bacteria but only for transcription and translation genes. Mol Microbiol 2006, 59:1506-1518.

21. Huvet M, Nicolay S, Touchon M, Audit B, d'Aubenton-Carafa Y, Arneodo A, Thermes $C$ : Human gene organization driven by the coordination of replication and transcription. Genome Res 2007, 17:1278-1285.

22. NCBI Organelle Genome Resources Website. [http://www.ncbi.nlm.nih. gov/genomes/OrganelleResource.cgi?opt=organelle\&taxid $=33208]$.

23. Seligmann H, Krishnan NM, Rao BJ: Mitochondrial tRNA sequences as unusual replication origins: pathogenic implications for Homo sapiens. $J$ Theor Biol 2006, 243:375-385.

24. Lynch M: The Origins of Genome Architecture Sunderland, MA: Sinauer 2007.

25. Shadel GS, Clayton DA: Mitochondrial DNA maintenance in vertebrates. Annu Rev Biochem 1997, 66:409-435.

26. Brown TA, Cecconi C, Tkachuk AN, Bustamante C, Clayton DA: Replication of mitochondrial DNA occurs by strand displacement with alternative 
light-strand origins, not via a strandcoupled mechanism. Genes Dev 2005, 19:2466-2476.

27. Saccone C, Pesole G, Sbisá E: The main regulatory region of mammalian mitochondrial DNA: structure-function model and evolutionary pattern. $J$ Mol Evol 1991, 33:83-91.

28. Xia X: Mutation and selection on the anticodon of tRNA genes in vertebrate mitochondrial genomes. Gene 2005, 345:13-20.

29. The CAIC User's Guide. [http://www.bio.ic.ac.uk/evolve/software/caic/index. html].

30. Sayle R, Milner-White EJ: RASMOL: biomolecular graphics for all. Trends Biochem Sci 1995, 20:374-376.

doi:10.1186/1471-2164-11-479

Cite this article as: Satoh et al:: Transfer RNA gene arrangement and codon usage in vertebrate mitochondrial genomes: a new insight into gene order conservation. BMC Genomics 2010 11:479.

\section{Submit your next manuscript to BioMed Central} and take full advantage of:

- Convenient online submission

- Thorough peer review

- No space constraints or color figure charges

- Immediate publication on acceptance

- Inclusion in PubMed, CAS, Scopus and Google Scholar

- Research which is freely available for redistribution

Submit your manuscript at www.biomedcentral.com/submit 